\title{
Entwicklung der Ordnungstherapie durch Bircher-Benner in der Naturheilkunde im 20. Jahrhundert
}

\author{
$\begin{array}{lll}\text { J. Melzera } & \text { D. Melcharta,b } & \text { R. Saller } \\ & \end{array}$ \\ a Departement Innere Medizin, Abteilung Naturheilkunde, Universitätsspital Zürich, Schweiz \\ b Zentrum für naturheilkundliche Forschung, II. Medizinische Klinik und Poliklinik, Klinikum rechts der Isar, \\ Technische Universität München, Deutschland
}

\section{Schlüsselwörter}

Ordnungstherapie · Bircher-Benner · Diätetik · Somatotherapie . Psychotherapie

\section{Zusammenfassung}

Hintergrund: Der Begriff der Ordnungstherapie stellt einen der fünf therapeutischen Bereiche dar, durch den die mitteleuropäische Naturheilkunde im deutschsprachigen Raum definiert ist. Fragestellung: Wer prägte den Begriff Ordnungstherapie in der Naturheilkunde, und welche Inhalte lassen sich herauskristallisieren? Methoden: Medizinhistorische Heuristik und Quellenkritik der Fachliteratur des 20. Jh. sowie Datenbankrecherche. Ergebnisse: In aktuellen deutschsprachigen medizinischen Lehrbüchern gehört die Ordnungstherapie zu den fünf therapeutischen Bereichen, die die mitteleuropäische Naturheilkunde definieren. Die inhaltliche Ausformulierung ist jedoch unterschiedlich und reicht von Gesundheitstraining bis zu körperorientierten Psychotherapieformen. Die Ordnungstherapie wird immer wieder Sebastian Kneipp zugeschrieben, Begriff und Konzeption stammen aber originär vom Schweizer Arzt Maximilian Bircher-Benner. Er prägt die Ordnungstherapie 1937 als Überbegriff für ein mehrdimensionales naturheilkundliches Therapiekonzept. Es basiert auf der nosologischen Vorstellung, dass Gesundheit Ordnung im menschlichen Organismus (physisch und psychisch), in der Umwelt und im Tagesablauf bedeute. Krankheit entstehe durch Unordnung in mindestens einem dieser Bereiche. Diese Ordnungstherapie ist inhaltlich durch neun Ordnungsgesetze bestimmt, die die Bereiche Ernährung, Hautorgan (Licht, Luft, Wasser), Atmung, Bewegung, Lebensrhythmus und Seelenleben umfassen. Bircher-Benner selbst gliedert die Ordnungstherapie in Somatotherapie (Ernährungstherapie, Sonnen- und Lichttherapie, Hydrotherapie, Bewegungstherapie, Atmungstechnik, Ordnung des Lebensrhythmus) und Psychotherapie. Diese therapeutische Konzeption geht auf die eklektische Auswahl komplementärmedizinischer Verfahren zurück, die er seit 1897 erlernte und nach seiner therapeutischen Empirie subjektiv für eine therapeutische Organisation auswählte. Gleichwohl sieht er die Ordnungstherapie auch im Zusammenhang mit gesellschaftspolitischen Kontexten der 1940er Jahre. Schlussfolgerungen: Der Terminus Ordnungstherapie wurde 1937 von Bircher-Benner als ein naturheilkundliches Therapiekonzept geprägt, das, bis auf Einschränkungen bei der Phytotherapie, die Bereiche umfasst, die in aktuellen Definitionen von Naturheilkunde genannt werden. Jedoch wird die Ordnungstherapie heute häufig als nur ein Bestandteil der Naturheilkunde (Ernähungstherapie, Hydrotherapie, Bewegungstherapie, Phytotherapie und Ordnungstherapie) angesehen, während sie bei Bircher-Benner ein mehrdimensionales therapeutisches Konzept darstellt. Die Einordnung der Ordnungstherapie als eine der "5 Säulen der Therapie nach Kneipp» stammt erst von Kneipp-Ärzten in der Mitte des 20. Jahrhunderts und ist daher neu zu diskutieren.

\author{
Key Words \\ 'Ordnungstherapie' · Bircher-Benner - Dietetics · Somatotherapy . \\ Psychotherapy · CAM - Life style change
}

\section{Summary}

Development of 'Ordnungstherapie' due to Bircher-Benner in the Naturopathy of the 20th Century

Background: The German term 'Ordnungstherapie' is one of the five therapeutics which defines naturopathy in German-speaking countries. Objective: Who formed the term Ordnungstherapie in naturopathy and what does it mean? Material and Methods: Heuristics and criticism of literature of the 20th century as well as database research. Results: Nowadays in German-language medical books Ordnungstherpie belongs to the five therapeutics which define European naturopathy. Yet, the interpretation ranges from health education to body-orientated forms of psychotherapy. The term Ordnungstherapie is often related with the German priest and hydropath Sebastian Kneipp, however, term and definition have been founded by the Swiss physician Maximillian Bircher-Benner. In 1937 he defined Ordnungstherapie as a complex concept of natural healing. It is based upon the rather nosological idea that health is order/harmony in the human body (physically, psychologically), the environment and the daily course. Illness occurs if disorder appears in one of these fields. The therapeutic setting of Ordnungstherapy is defined by 9 rules of conduct to maintain order, which include nutrition, the skin as an organ (exposure to light, air, water), breathing, movement, rhythm of life, and psyche. For all these aspects Bircher-Benner himself uses the terms somatotherapy (dietotherapy, sun and light therapy, hydrotherapy, exercise therapy, breathing technique, order of the rhythm of live) and psychotherapy. He chose these complementary methods subjectively after learning them from 1897 onwards in an eclectic manner and after gaining therapeutic empiricism. Nevertheless his ideas of the Ordnungstherapie correlate with the socio-political context of the 1940ies. Conclusions: The term Ordnungstherapie was introduced by Bircher-Benner as an umbrella term in 1937 to describe a complex concept of naturopathic therapies. It comprises, with certain limitations for phytotherapy, the therapies which nowadays define European naturopathy. Yet, in European naturopathy today Ordnungstherapie is mostly considered as one out of 5 constituents of naturopathy (dietotherapy, hydrotherapy, exercise therapy, phytotherapy, Ordnungstherapie). The classification of Ordnungstherapie as one of the 5 pillars of the Kneipp therapy was only done by Kneipp physicians in the middle of the 20th century and needs to be thought over.

\begin{tabular}{ll}
\hline KARGER & @ 2004 S. Karger GmbH, Freiburg \\
$\begin{array}{l}\text { Fax +497614520714 } \\
\begin{array}{l}\text { E-mail Information@Karger.de } \\
\text { www.karger.com }\end{array}\end{array}$ & $\begin{array}{c}\text { Accessible online at: } \\
\text { www.karger.com/fkm }\end{array}$ \\
&
\end{tabular}




\section{Einleitung}

Der historische Begriff der Ordnungstherapie stellt in aktuellen deutschsprachigen Lehrbüchern der Naturheilkunde einen der fünf therapeutischen Bereiche dar, die das Gebiet der mitteleuropäischen Naturheilkunde definieren: Hydrotherapie, Bewegungstherapie, Ernährungstherapie, Phytotherapie, Ordnungstherapie [1,2]. Darüber hinaus gehört er zu den Inhalten der Weiterbildungsordnung für Ärzte in Deutschland (Zusatzbezeichnung Naturheilverfahren) [z.B. 3].

Die inhaltlichen Beschreibungen der Ordnungstherapie variieren jedoch je nach Autor. Gemeinsam ist vielen Autoren, die Ordnungstherapie als eine Intervention zur Schaffung von Lebensordnung zu sehen. Die einen betonen dabei mehr psychologische, die anderen mehr körperbezogene naturheilkundliche Massnahmen (Tab. 1). Daher muss auch heute noch bei jedem Autor hinterfragt werden, welche Inhalte er meint, wenn er von Ordnungstherapie spricht. Meist wird die Ordnungstherapie in Zusammenhang mit den so genannten fünf Therapieprinzipien oder Säulen nach Sebastian Kneipp (1821-1897) bzw. der Kneipp-Therapie genannt. Eindeutige Quellennachweise für die Prägung des Begriffes durch Kneipp fehlen bisher. Erst R. Wilhelm wies 1990 auf die Prägung des Begriffes Ordnungstherapie durch Bircher-Benner hin [4], und einige Autoren folgten seinen Ausführungen partiell $[5,6]$.

Diese unklare Situation in Bezug auf Inhalt und Ursprung der Ordnungstherapie dürfte ein Grund sein, warum sich in medizinischen Datenbanken (MEDLINE, old MEDLINE, BIOSIS, PsycINFO, CDSR, ACP Journal Club, DARE, CCRCT, CINAHL, vom jeweiligen Beginn bis 2. Woche 2004, Suchbegriff: Ordnungstherapie) nur ein Artikel findet, in dem der Begriff kurz im Rahmen der Definition der Kneipp-Therapie genannt wird. In den derzeitigen EBMorientierten Ansätzen, wichtige Gebiete der Medizin zu evaluieren, kommt das im Rahmen der Naturheilkunde regelmässig genannte «Therapiekonzept» nicht vor. Das Fehlen eines theoretisch wie auch praktisch konstituierenden Konzeptes der Naturheilkunde weist auf eine erhebliche Authentizitätsproblematik einengender Evaluationsmethodologie hin. Im Folgenden werden daher Entwicklung und Inhalte des therapeutischen Konzepts der Ordnungstherapie nach Bircher-Benner dargestellt.

\section{Frühe biographische Aspekte der Ordnungstherapie bei Bircher-Benner}

In der Biographie von Maximilian Bircher-Benner (18671939) finden sich einige Hinweise darauf, dass das Instruieren zu einem wichtigen strukturierenden Element in seinen ersten beiden Lebensjahrzehnten wurde. Von seiner Jugend, die geprägt war von finanziellen Schwierigkeiten und schliesslich dem Verlassenwerden von seinem Vater, einem Notar, schreibt er: «An meinen Klavierschülern lernte ich die Anfänge der Menschenführung, an den Artilleristen aber das Instru- ieren und Kommandieren einer grösseren Menschengruppe» [7, S. 21]. In diese Zeit fallen auch seine ersten Erfahrungen mit der Hydrotherapie, als er wegen familiärer Probleme an Schlaflosigkeit leidet und von seinem Reitlehrer, einem ehemaligen Artilleristen, mit Erfolg die Wirkung von PriessnitzWickeln lernt [ibid., S. 23].

Als Bircher-Benner während seines Medizinstudiums in Zürich abermals an Schlafstörungen leidet, strukturiert er seinen Tagesablauf nach einem strikten Schema: «Abends 5 Uhr ... marschierte ich .... zwei Stunden ... nahm ein frugales Abendmahl und legte mich um 8 Uhr ins Bett. Um 4 Uhr 30 stand ich auf, machte auf dem Balkon Atemübungen, .. studierte ... [ging] in die Vorlesung.» Er selbst resümiert, «gesunde Lebensordnung und ein bisschen heroische Selbstbeherrschung» hätten ihm damals den Schlaf wiedergebracht [ibid., S. 25]. Das Ordnen des Tagesablaufs scheint von einer Idee geprägt zu sein, die Bircher-Benner durch die Physiologievorlesungen entwickelt: Die Physiologie, als «die Lehre vom Leben», lies bei ihm «Vorstellungen vom Reiche der Ordnungen im Lebendigen» entstehen, wobei «Gesundheit ein wohlgeordnetes Leben voraussetzt, ... jegliche Unordnung [aber] zu Gesundheitsstörungen und Krankheiten führt» [ibid., S. 27 f].

\section{Bircher-Benner erlernt komplementärmedizinische Methoden} Noch während seines Studiums in Zürich lernt Bircher-Benner die Hypnosebehandlung von dem Psychiater August Forel (1848-1931) kennen, seinem Hochschullehrer und Betreuer seines ersten Dissertationsversuches. Durch ihn wird er auch auf die Lehre der Mneme nach dem Biologen Richard Semon (1859-1918) aufmerksam, die später sein Verständnis von Psychotherapie nachhaltig prägt [ibid., S. 33]. Als Unterassistent erfährt er vom Leiter der gynäkologischen Poliklinik die Thure-Brandt-Massage, und anhand der «Klinischen Blätter für Hydrotherapie» beginnt er, die Kneipp-Therapie zu lernen [ibid., S. 37 f].

In den Jahren des Aufbaus einer eigenen Praxis in Zürich, ab 1891, berichtet Bircher-Benner nichts über naturheilkundliche Methoden. Im Jahr 1895 wird er dann auf die Rohkosternährung aufmerksam [7, 8] und im Jahr 1897 unterbricht er seine Praxistätigkeit, um bei mehreren Naturheilkundlern Einblick in deren Therapien zu gewinnen. Er hospitiert in der hydropathischen Anstalt bei Wilhelm Winternitz (1834-1917) in Wien, lernt dort bei Bumm auch Massagetechniken kennen und liest Schriften von Sigmund Freud. Von Österreich reist er nach Dresden in das Sanatorium «Weisser Hirsch» von Heinrich Lahmann (1860-1905), um einen Einblick in dessen Diätetik, Licht- und Luftbäder zu erhalten. Schliesslich trifft er sich noch mit dem Physiologen Max Rubner in Berlin und bespricht Fragen der Ernährung. Als Bircher-Benner im gleichen Jahr wieder nach Zürich zurückkehrt, eröffnet er eine kleine Privatklinik in der Asylstrasse am Zürichberg sowie ein Institut für Hydrotherapie und physikalische Heilmethoden und beginnt, die erlernten naturheilkundlichen Methoden umzusetzen. 


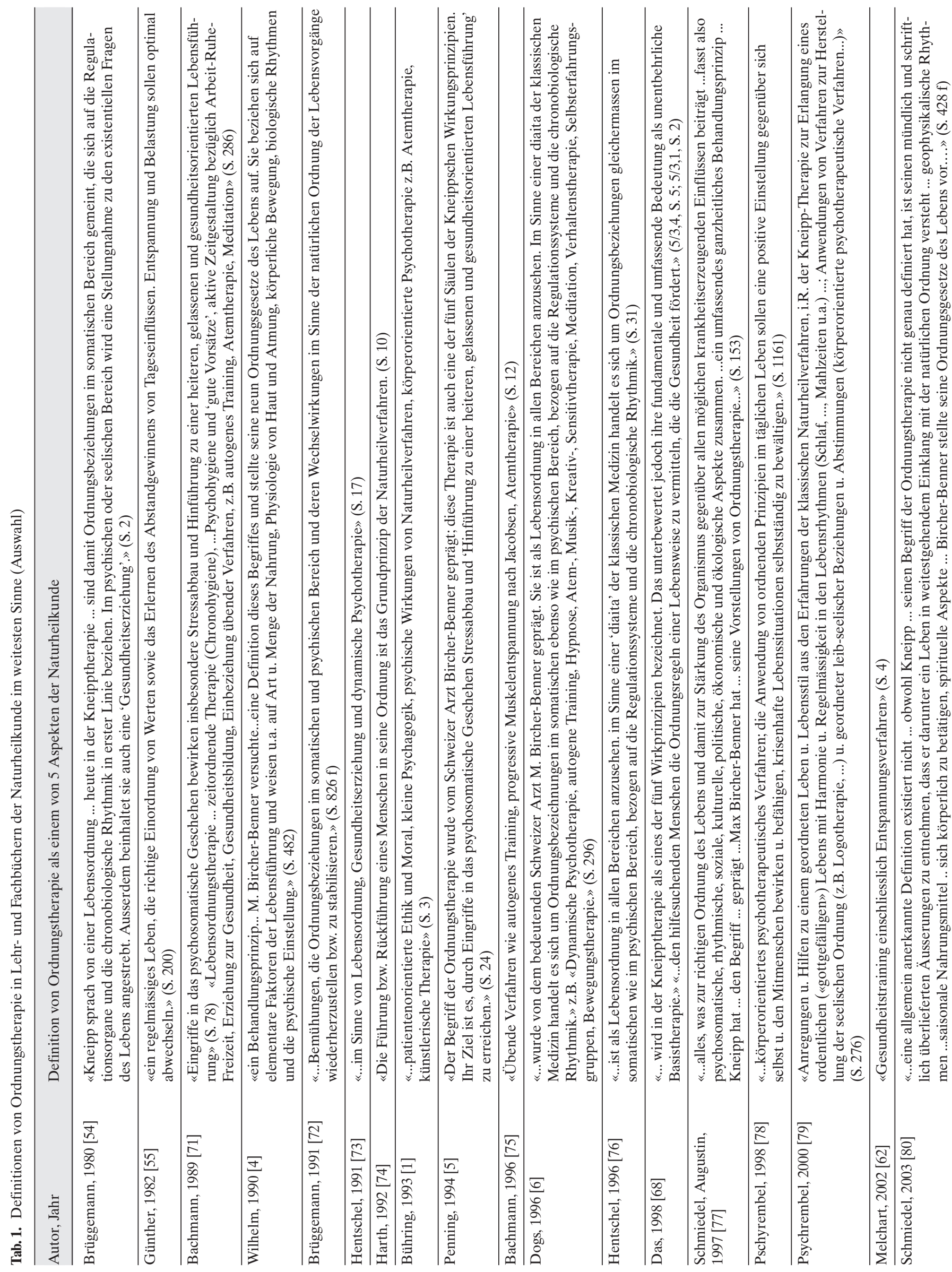


Tab. 2. Spektrum der Therapieverordnungen von Bircher-Benner aus den 1910 bis 1930er Jahren [81] (Auswahl)

\begin{tabular}{|c|c|c|}
\hline Wickel, Güsse, Bäder, Massagen & Ernährung & Bewegung, Beschäftigung \\
\hline $\begin{array}{l}\text { Waden-, Leib-, Antiphlogestinwickel, } \\
\text { Bein-, Rücken-, Oberkörper-, Unterkörper-, Ganzwaschung, } \\
\text { Knie-, Voll-, Schulterguss, Kopfbegiessung, Fuss-, Knie-, Schenkel-, } \\
\text { Arm-, Sitz-, Halb-, Reibesitz-, Regen-, Schwitz-, Dampf-, } \\
\text { Heublumenbad, Sprudelbad m. Eukalyptus, Wassertreten, } \\
\text { Luft-, Dauerluftbad, Sonnenbad, Bett-Glühlicht, Bettlichtbad, } \\
\text { Höhensonne bis zu leichtem Schwitzen, } \\
\text { Heissluftkasten, Massage, Körpermassage }\end{array}$ & $\begin{array}{l}\text { Mehlmüsli mit Milch, } \\
\text { geriebener Apfel, } \\
\text { Mandelmilch, } \\
\text { passiertes Gemüse, } \\
\text { knappe Vollkost, } \\
\text { strenge Rohkost, } \\
\text { Roh-, Übergangs-, Normalkost }\end{array}$ & $\begin{array}{l}\text { Frühmarsch und Gymnastik im } \\
\text { Luftbad, Gymnastik im Saal, } \\
\text { Turnen, Morgenspaziergang, } \\
\text { Spaziergang, Atemgymnastik, } \\
\text { Schwimmbäder, Frühritt, } \\
\text { Rudern, Gartenarbeit, } \\
\text { Ruhen auch im Garten }\end{array}$ \\
\hline
\end{tabular}

\section{Komplementärmedizinische Erfahrungen, eigene}

\section{Theoriebildung und Therapiegestaltung}

Als er rund sieben Jahre später, 1904, sein Sanatorium «Lebendige Kraft» am Zürichberg eröffnet, formuliert BircherBenner für einen Prospekt die ersten eigenen Therapievorschriften. Sie lassen die Strukturierung des Tagesablaufes nach einem ordnenden Prinzip erkennen. So sei es etwa ratsam, unter Beachtung der zirkadianen Tagesrhythmik, «vor allem nach Ordnung, Regelmässigkeit und Pünktlichkeit zu streben. Der Ablauf der Lebensvorgänge beim Gesunden während 24 Stunden des Tages hat einen ausgesprochenen regelmässigen periodischen Verlauf.» Da er diese Periodizität beim Kranken als gestört ansieht, sei die «Durchführung eines geregelten Lebens» die Voraussetzung zur Gesundung [9] und daher eine klare «Tagesordnung» notwendig.

In seinem seit 1903 erscheinenden Ernährungsbuch findet sich ebenso die Umsetzung einer Ordnung, diesmal auf dem Gebiet der Ernährung. Aufgrund Bircher-Benners energetischer Auffassung der Ernährungslehre, Pflanzennahrung enthalte eine Art gespeicherter Sonnenenergie, stellt er eine Ordnung der Nahrungsmittel nach «Akkumulatoren erster bis dritter Ordnung» vor [10-14; 15, S. 15]. Um dem Vorwurf der Einseitigkeit vorzubeugen und einen therapeutischen Rahmen aufzuzeigen, weist er am Ende des Buches seiner Ernährungstherapie den Platz als «Glied in dem Gesamtorganismus der Therapie» zu [ibid., S. 244]. Er sagt, die «Einheit der Ernährung [sei] nicht der Organismus [alleine] sondern Organismus, Nahrung und Lebensraum.»

Seine Auffassung von den Begriffen Organismus und Lebensraum führt er nur recht kryptisch aus: Die «Gesamtheit der Eigenschaften des Organismus nennt man Konstitution» [ibid., S. 246] und eine «Organisationstherapie» sei notwendig, um «den Weg zur konstitutionellen Kräftigung der nachfolgenden Generationen zu zeigen» [ibid., S. 248].

Unter Lebensraum versteht er die Wirkungen der physikalischen Kräfte auf den Körper, die geregelt, als «physikalische Therapie», zum Ausgleich genutzt werden könnten [ibid., S. 249]. Ausserdem wirkten im Lebensraum die vielfältigen «psychischen Wirkungen» des Lebens, die durch «Psychotherapie» auszugleichen seien [ibid., S. 252 f]. Die physikalische Therapie und die Psychotherapie fasst er unter dem Begriff der «Therapie der Arbeit» zusammen und subsumiert darunter auch «Beschäftigungstherapie und Terrainkur» [ibid., S. 253].
Nach dieser kurzen Umreissung der drei für ihn wichtigen therapeutischen Bereiche resümiert er: Der «Gesamtorganismus der Therapie» umfasse «die Organisationstherapie, die Ernährungstherapie und die Therapie der Arbeit» [ibid., S. 254].

Der Rahmen der praktischen Umsetzung des «Gesamtorganismus der Therapie» wird an den «Kurvorschriften» erkennbar, die den stationären Patienten von Bircher-Benner ausgehändigt wurden. In einem Exemplar aus dem Jahre 1925 ist unter «Einführung in das Leben des Sanatoriums» zu lesen: Das Sanatorium schafft «ein Milieu für den Kranken», wo ihm als «Suchenden ...viele Lebensregeln offenbart» werden. Vom ihm wird aber erwartet, dass er sich «in das Leben des Sanatoriums einfügt und die Anordnungen gewissenhaft ausführt». Dann wird er, beim «Eindringen in den Heilgedanken des Sanatoriums», vom Personal unterstützt und die ganze Kur unterliegt fortan der «ärztlichen Überwachung» [16]. An dieser Skizzierung des therapeutischen Rahmens wird auch die von Bircher-Benner geforderte Schaffung einer «Umwelt, eines Milieus» für den Kranken im Sinne einer «Milieutherapie» verständlich [17, S. 242; 18, S. 491].

Neben den Kurvorschriften werden, vor allem durch die Therapieverordnungen von Bircher-Benner, eindrückliche Angaben über das Spektrum der angewandten Therapien deutlich, auch wenn die nur noch teilweise vorhandenen Akten seiner stationären Patienten keinen repräsentativen Überblick ermöglichen (Tab. 2). In diesen Akten verordnet er pro Patient ca. 3-6 Therapien, was aber nach Erkrankung, Alter, Jahreszeit schwanken konnte.

\section{Theorie der "therapeutischen Organisation»}

Erst nach Jahren ärztlicher Tätigkeit, mit Erweiterung der Privatklinik, Gründung eines Verlages und einer Zeitschrift und mehreren Buch- und Zeitschriftenpublikationen, stellt Bircher-Benner 1935 ein überarbeitetes und erweitertes Therapiekonzept vor. In dem Buch «Vom Sinn einer therapeutischen Organisation» [15] beschreibt er, wiederum ausgehend von den Vorstellungen aus der Physiologievorlesung, dass er mittlerweile selbst «ein Milieu, eine Heilstätte, in der eine ärztliche optimale Lebensordnung den Kranken ihre Gesundheit wiederbringe», geschaffen habe. Die Grundlage der Verwirklichung dieser «therapeutischen Organisation» stellt für ihn die «ursächliche Therapie» dar [ibid., S. 5; s.a. 19]. Dazu äussert er sich folgendermassen: Die Ursachen der «verbrei- 
tetsten Krankheiten» liegen «in der Lebensführung, insbesondere jener Lebensführung, die sich unter der Industrialisierung des Lebens, unter dem Einfluss der Zivilisation, im Laufe der letzten Jahrzehnte herausbildete.» Und er erklärt dazu: «An die unrationelle Ernährung der zivilisierten Nationen reihen sich als weitere Krankheitsursachen naturwidrige Tagesordnung, ungenügende Hautpflege und übergrosse seelische Beanspruchung.» All diese Krankheitsursachen führen für ihn zu einer zunehmenden «Konstitutionsverschlechterung» des Menschen [ibid., S. 6].

Aus therapeutischer Sicht sei es aber möglich, «im Lebenden» wieder $\mathrm{zu}$ einem «normalen Gleichgewicht zurückzukehren» und eine Wiedergenesung zu erreichen, «solange die Erkrankung jene gewisse Schwelle noch nicht überschritten» habe. Dann nämlich könne die «Naturheilkraft ... die Gesundheit wiederherstellen», wenn die Ursache überwindbar ist oder durch ärztliche Massnahmen behoben werden könne. Da die Krankheitsursachen hauptsächlich «in der Lebensführung» lägen, müssten die «Heilmassnahmen des Arztes» dort ansetzen [ibid., S. 7]. Eine ursächliche Therapie sei nur möglich, wenn «Speise und Trank, ...Arbeit und Ruhe ... Wachsein und Schlaf, ... Pflege des Hautlebens und seelisches Erleben ... mit den Ordnungsgesetzen des Lebens in Einklang» gebracht würden [ibid., S. 8; s.a. S. 20]. Die Aufzählung dieser fünf Bereiche zeigt eine Parallele zu den sechs Aspekten in der antiken Diätetik, die Galen in Folge der pythagoreischen und hippokratischen Diaita nannte [14, S. 41]. Und in der Tat bezieht sich Bircher-Benner im Sinne historischer Verweise auf Hippokrates, aber er ist stärker von zeitgenössischen Autoren beeinflusst [15, S. 14; 7, S. 10 , 12]. So bezieht er sich bei der «kausalen Therapie» auf die Ausführungen des Ordinarius für Medizin in Riga, Martin Sihle [15, S. 9, s.a. 20], von dessen Buch er 1934 so beeindruckt ist, dass er einen kleinen Auszug in einer eigenen Schrift herausgibt [21]. Die Beeinflussung durch Sihle wird auch daran deutlich, wie Bircher-Benner sein Buch über die «therapeutische Organisation» in Anlehnung an drei Kernbegriffe von Sihle gliedert: a. die «ursächliche Therapie», b. «die Pflege des Blumenbeetes» und c. «die Pflege des Seelenlebens». Der Begriff «Blumenbeet» stellt bei Sihle eine Metapher für das mesenchymale Grundgewebe im menschlichen Organismus dar [20, S. 113]. Er stützt sich dabei auf Ausführungen des Arztes Felix Buttersack (1865-1950), die später auch von anderen, z.B. Alfred Pischinger (1899-1982), aufgegriffen wurden.

Für Bircher-Benner liegt in den weiteren Ausführungen der «Schwerpunkt in der ,Pflege des Blumenbeetes'» durch Heilernährung, die in der «ursächlichen Therapie die conditio sine qua non» sei, da sie die «mächtigste Ursache von Gesundheitsstörungen» darstelle. Ein wichtiges «natürliches Ordnungsgesetz der Ernährung» ist für ihn die «natürliche Korrelation sämtlicher Nährfaktoren» im naturbelassenen Nahrungsmittel [ibid., S. 11]. Ausserdem sei bei der Ernährung auf «richtiges Mass und Ordnung», im Sinne des Nahrungsbedürfnisses des Körpers in Abhängigkeit vom Kräfteverbrauch, zu achten [ibid., S. 12].
Schliesslich zählt er neben der Heilernährung noch acht weitere «Heilmassnahmen» auf, die in der «somatischen Therapie» nützlich seien und wählt dafür die Worte: 1. «die Lebensordnung ... , 2. die Pflege des Hautlebens (Luftbäder, Bäder, Güsse, Packungen, Waschungen, Lichtbäder) ..., 3. Atempflege ..., 4. Heilgymnastik ... , 5. angemessenes Gehen und Marschieren im Freien ..., 6. Arbeitstherapie (zielgerichtete Beschäftigung) ..., 7. Anwendung der Elektrizität (galvanischer, faradischer Strom, Hochfrequenz, Diathermie) ..., 8. allgemeine und örtliche Massage» [ibid., S. 20 f].

Dass dieser Rahmen der Heilmassnahmen für Bircher-Benner keine absolut geschlossene Form darstellt wird deutlich, wenn er beispielsweise auf die Entwicklung der «Kräuterheilkunde, der Mineralisationsfrage, der Homöopathie, der Chirurgie, der Frauenheilkunde, ... der Behandlung mit ozonisiertem Sauerstoff und der Chiropraktik» verweist. Das grosse Spektrum der «somatotherapeutischen Organisation» ermögliche weitgehend einen Verzicht auf «die symptomatische Behandlung mit Medikamenten, Präparaten, Seren und Vakzinen», aber er betont: «Weitgehend heisst nicht gänzlich.» [ibid., S. $22 \mathrm{f}$ ]

In den Ausführungen zur «Pflege des Seelenlebens» unterstreicht Bircher-Benner, dass sich «Somatotherapie und Psychotherapie zu einer Einheit» vereinigen müssten. Auch er spricht von der seit den 1920er Jahren erwähnten «Leib-SeeleGeist-Einheit» des Menschen. Zum Geist macht er allerdings keine Ausführungen, da er nicht erkranken könne. Zur Seele sagt er, ihre «Kraft» sei bedingt «1. durch das Erbe ..., 2. durch den Ernährungsstrom ... und 3. durch die Empfangsfähigkeit für die Einströmung geistiger Energien.» Da «in jeder Krankheit Körperliches und Seelisches gemischt», aber in unterschiedlichen Anteilen erkrankt seien, müsse für den Körper («funktionell und organisch») wie auch für die Psyche eine Diagnose und Behandlung erfolgen [ibid., S. 24 f]. Der Kranke habe sodann die Diagnose zu erfahren, damit er nicht vom Arzt, sondern «von seinem eigenen Verhalten gegenüber einem inneren Ordnungsgesetz des Lebens» die Heilung erwarte [ibid., S. 26]. Ferner sei zu beachten, wie der Kranke oft durch ein «Spinnengewebe von Beziehungen mit seiner Umwelt verbunden» sei, was die Heilung verhindern könne. Daher verlange «die Heilung eine andere ..., eine organisierte Umwelt», wie z.B. in einer Klinik [ibid., S. 28]. Dort könne sich der Kranke wieder dem «Reich der Lebensordnungen» nähern, da die «Synthese aller Heilmassnahmen ... [die] zielgerichtete Ordnung der Lebensbedingungen» ermögliche [ibid., S. 31].

Diese Ausführungen verdeutlichen Kernaspekte des nosologischen (Krankheit gleich Unordnung) und therapeutischen (Somato- und Psychotherapie) Verständnisses von BircherBenner sowie des therapeutischen Rahmens (Schaffung eines Milieus bzw. einer Klinik).

\section{Konzeption der Ordnungstherapie}

Erst zwei Jahre vor seinem Tod, 1937, verdichtet Bircher-Benner seine therapeutische Methode begrifflich zur «Ordnungs- 


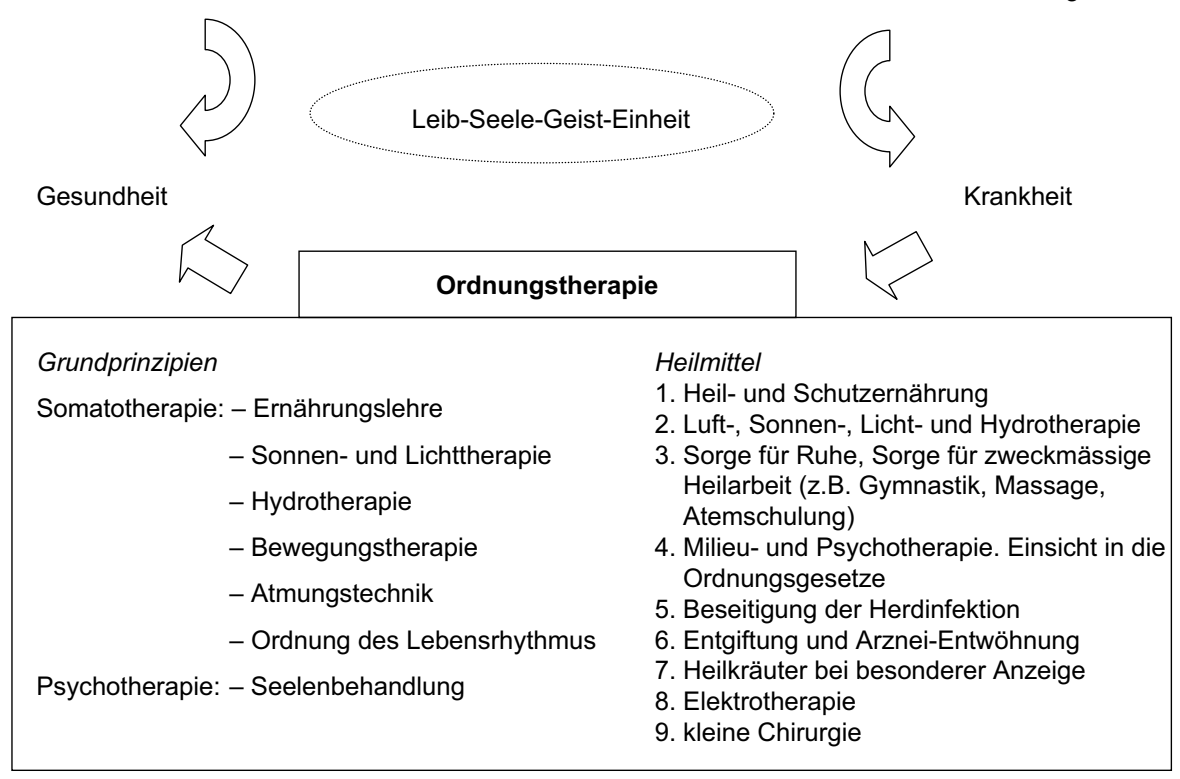

Abb. 1. Begriffe und Konzeption der Ordnungstherapie nach Bircher-Benner [22, S. 125, 133 f; 83, S. 22] pie in Therapiegruppen zusammen. Auf dem Gebiet der Psychotherapie hält er, vor Beginn der «individuellen Psychotherapie», «die Ordnung der Vorstellungen des Kranken über seine Krankheit» durch eine klare Diagnosestellung samt deren Erklärung und «die Organisation der Umwelt des Kranken» für notwendig [ibid., S. 136 f]. An der Unterteilung der Ordnungstherapie in Somatotherapie und Psychotherapie, mit ihren jeweiligen Therapieformen, wird seine Sichtweise dieser therapeutischen Organisation als einer umfassenden naturheilkundlichen Behandlung deutlich (Abb. 1).

Nachdem Bircher-Benner bereits im Jahr 1933 Vorträge vor der «Food Education Society» von Sir Robert McCarrison in London gehalten hatte, spricht er dort abermals im Oktober 1937. In diesen Vorträgen erklärt er auch sein Konzept der Ordnungstherapie und formuliert darin neun Ordnungsgesetze, die zur Erhaltung der Gesundheit - oder beim Vorliegen von «Unordnungskrankheiten» zur Gesundung - beachtet werden sollen (Tab. 3).

Mit diesen Ordnungsgesetzen benennt Bircher-Benner gleichsam neun Regeln, die der Einzelne beachten soll, um sich gesund zu halten oder wieder gesund zu werden. Die Ausformulierung dieses Therapiekonzepts der Ordnungstherapie und der Ordnungsgesetze, die abermals Körper und Psyche umfassen, können als reflektierter Abschluss des ärztlichen Wirkens von Bircher-Benner verstanden werden. Das Konzept ist für ihn selbst so überzeugend, dass er es auch als «Therapia magna» bezeichnet [7, S. 111, 181; 22, S. 95] und am Ende seiner Biographie ein eigenes Kapitel der «Ordnungstherapie» widmet [7].

\section{Popularisierung der Ordnungstherapie}

Noch zu Lebzeiten ermöglicht Bircher-Benner eine Verbreitung des Konzeptes der Ordnungstherapie. Seine Privatklinik «Sanatorium Lebendige Kraft» galt, von regelmässigen Ausnungstherapie und fasst einzelne Aspekte der Somatothera- 
Tab. 3. Die 9 Ordnungsgesetze der Ordnungstherapie nach Bircher-Benner [82, S. 19-33, 116]

\begin{tabular}{lll}
\hline Nr. & Ordnungsgesetz & Inhalt \\
\hline 1 & «Das Organisationsgesetz der Nahrung» & $\begin{array}{l}\text { Ein unverändertes pflanzliches Nahrungsmittel stellt ein «Integral» an } \\
\text { Nährfaktoren dar. }\end{array}$ \\
\hline 2 & «Das Gleichgewichtsgesetz der Ernährung» & $\begin{array}{l}\text { Im Nahrungsintegral liegen die Nährfaktoren in einer «natürlichen Korrelation» } \\
\text { vor. Mit beiden Gesetzen unterstreicht Bircher-Benner die Ansicht, von } \\
\text { Nahrungsmitteln keine Bestandteile unnötigerweise abzutrennen. }\end{array}$ \\
\hline 3 & «Das Ökonomiegesetz» & Mahnt zu einer ausreichenden aber knappen Nahrungszufuhr. \\
\hline 4 & «Das Mundgesetz» & Bezieht sich auf das ausreichende Kauen der Nahrung. \\
\hline 5 & «Das Ordnungsgesetz des Hautorgans» & $\begin{array}{l}\text { Betrifft unterschiedliche Aspekte, wie den Kontakt zum Sonnenlicht zur beginnen- } \\
\text { den Vitamin-D-Bildung in der Haut, aber auch die Wirkung von hydrotherapeuti- } \\
\text { schen Anwendungen auf die Haut als Regulationsorgan. }\end{array}$ \\
\hline 6 & «Das Ordnungsgesetz der Lungen» & Betont dass frische, reine Luft zum Atmen vorhanden sein soll. \\
\hline 7 & «Das Ordnungsgesetz der Beziehung zur Schwerkraft» & $\begin{array}{l}\text { Steht für die dem jeweiligen Gesundheits- und Alterszustand angemessene } \\
\text { körperliche Betätigung. }\end{array}$ \\
\hline 8 & «Das Ordnungsgesetz des Lebensrhythmus» & $\begin{array}{l}\text { Umfasst die tages- und jahreszeitliche Periodik, wie auch die Beachtung der für } \\
\text { Bircher-Benner besonders effektiven «Naturschlafzeit» (19:30-24:00 Uhr) nach } \\
\text { T. Stöckelmann. }\end{array}$ \\
\hline 9 & «Das Ordnungsgesetz des Seelenlebens» & $\begin{array}{l}\text { Beinhaltet die Einwirkungen aller psychischen Aspekte auf den Menschen und } \\
\text { gegebenenfalls ihre Bewältigung mittels Psychotherapie. }\end{array}$ \\
\hline
\end{tabular}

nahmen abgesehen, eher als Refugium für wohlhabendere Patienten. Doch die praktische Anwendung der Ordnungstherapie dort und deren Popularisierung in Vorträgen, Zeitschriftenartikeln und Büchern sah er nicht als ausreichend an. Die Spende eines Patienten über 1 Million Franken ermöglicht ihm, 1937 zu verkünden, ein «Volkssanatorium für Ordnungstherapie» errichten zu wollen [24, S. 244]. An seinem 70. Geburtstag im gleichen Jahr erhält Bircher-Benner ein Geschenk von 5000 Franken mit der Widmung, dass dies das Anfangskapital für einen «Freibettfond» für das neue Sanatorium sein solle [19, S. 529].

Entscheidenden Anteil an der Realisierung des Volkssanatoriums hat die 1935 gegründete «Zürcher Kulturgesellschaft» (ZKg), in der Bircher-Benners Sohn Ralph (1899-1990) mitarbeitet und dessen Organ die Zeitschrift «Der Wendepunkt» ist. Bircher-Benner und seine Söhne halten Vorträge in der ZKg, und er selbst wiederholt dort seine Londoner Vorträge im Dezember 1937 und stellt somit die Ordnungstherapie in Zürich vor [28]. Mit der Neugründung der Kulturgesellschaft Zürich (KgZ), 1938 als gemeinnütziger Verein, übernimmt sie das Protektorat für das «Dr. Bircher'sche Volkssanatorium» [29]. Franklin Bircher (1896-1988) wird Präsident der Gesellschaft und später auch Chefarzt des Volkssanatoriums.

Im April 1938 erwirbt die zwischenzeitlich gegründete Stiftung für das Volkssanatorium, dessen Präsident Max Edwin Bircher ist, am Zürichberg das ehemalige Haus und Gründstück der Familie Kissling, nicht weit von der Privatklinik entfernt [30]. Der «Freibettenfond», der besonders Hilfebedürftigen eine kostenlose Behandlung ermöglichen soll, ist auf 8000 Franken angewachsen und beträgt Mitte 1939 bereits 37000 Franken. Zusätzlich wird ein «Wissenschaftlicher Fond» eingerichtet, der dazu dienen soll, «Forschungsergebnisse und
Arbeiten auf dem Gebiet der Ordnungstherapie» zu veröffentlichen [31].

Noch Bircher-Benner selbst inseriert, dass er einen «jüngeren Schweizerarzt» suche, der über einen Stiftungskredit eine 2-jährige Ausbildung in der Privatklinik erhält, um sich für die Arbeit im Volkssanatorium vorzubereiten [32].

Bircher-Benner erlebt die Eröffnung des Volkssanatoriums im Juni 1939 nicht mehr. Doch sein Sohn Franklin Bircher setzt sein Konzept im Volkssanatorium um und wertet die Ergebnisse der ersten 100 Patienten aus [33]. Sein Sohn Willy Bircher publiziert seine Beobachtungen über die Wirkung der Ordnungstherapie bei Herzkrankheiten und Hautkrankheiten [34, 35].

Die Nichte und spätere Chefärztin der Privatklinik, Dagmar Liechti von Brasch (1911-1993), veröffentlicht 1970 einen Artikel über «70 Jahre Erfahrungsgut der Bircher-Benner Ordnungstherapie», wobei sie nur kurz acht Aspekte der Ordnungstherapie nennt, um sich dann auf die Ausführungen zu den ersten drei Ordnungsgesetzen zu beschränken sowie von Erfahrungen mit der Rohkosttherapie zu berichten [36].

\section{Gesellschaftspolitische Aspekte der Ordnungstherapie bei Bircher-Benner}

Bereits Wirz hatte 1993 darauf aufmerksam gemacht, dass Bircher-Benner «eine biologische Weltsicht» sowie ein «patriarchalisch-autoritäres Politikverständnis mit einem ,Führer als oberster Instanz» habe [37, S. 142]. Die «biologische Weltsicht» zeigt sich früh in Bircher-Benners Veröffentlichungen. So warnt er schon 1909 im Zusammenhang einer ungesunden Ernährung vor «Schwächung der Konstitution, Störung des Stoffwechsels, grosser Anfälligkeit für Infektionen, kurz eine Verschlechterung der Gesundheit und einer Degeneration der 
Rasse» [10, S. 23; s.a. 15, S. 10]. Die mögliche Verbesserung der Konstitution sah er damals durch «Zuchtwahl» gegeben, die durch «Organisationstherapie» eine «Rassenverbesserung» bringe, da sie «den Weg zur konstitutionellen Kräftigung der nachfolgenden Generationen zeige» [ibid., S. 247 f]. Mit seinen Ausführungen zur Konstitutionsverschlechterung und Rassendegeneration [s.a. 14, S. 134-139] befindet sich Bircher-Benner im Rahmen der Aussagen der eugenischen Bewegungen, die in den 1920er Jahren weltweit zu finden sind [38]. Er scheint aber keiner Konstitutionslehre besonders anzuhängen, auch wenn er Alfred Mac Cann in diesem Zusammenhang häufig als Autor zitiert.

Bircher-Benner mahnt vor «angeborener minderwertiger Anlage und Konstitution» [22, S. 134] oder «körperlichen und seelischen Minderwertigkeiten», wobei meist der genealogisch-völkische Aspekt überwiegt. So spricht er davon, dass sich ein Leben wider den Ordnungsgesetzen von den «Sünden der Väter [an] bis ins dritte und vierte Glied» räche [22, S. 122]. Meist ist dies verbunden mit einem starken erzieherischen Impetus, wobei er Schuld und Sünde nicht nur beim Einzelnen sieht, sondern auch von der «Schuld der ganzen Menschheit» spricht [15, 28]. Als ein Student von Forel, der radikale Forderungen und Praktiken bis zur Zwangssterilisierung erhob und umsetzte, scheint Bircher-Benner, in der umfangreich gesichteten Literatur [14], nicht so weit zu gehen. Gleichwohl zeichnet sich in Buchbesprechungen rassenhygienischer Literatur im Wendepunkt ein Sympathisieren mit eugenisch-rassenhygienischen Aspekten ab [14]. Anstatt eigene klare Aussagen zu machen, zitiert Bircher-Benner gerne andere, wie etwa am Beginn seiner Biographie Nietzsche mit den Worten «...ein guter Arzt ... ist dann imstande, der ganzen Gesellschaft ein Wohltäter zu werden ... durch Herstellung einer geistig-leiblichen Aristokratie (als Ehestifter und Eheverhinderer)» [7, S. 6]. Zeitgleich mit den ersten Mahnungen vor Konstitutionsverschlechterung unterstreicht Bircher-Benner, dass für ihn eine entsprechende Therapie «eine Aufgabe [darstelle], welche nicht durch den einzelnen Arzt, sondern nur durch die Gesamtheit der Ärzte, in Verbindung mit Familie, Schule und Staat gelöst werden kann» [10, S. 247]. Er wird nicht müde, eine Ausbildungsstätte für Ärzte und die staatliche Unterstützung einer solchen Einrichtung zu fordern. Er meint dann, in Mussolinis «naturismo» und in der «Neuen Deutschen Heilkunde der Nationalsozialisten» das korrekte Handeln von Staatsführern zu sehen, die eine Gesundheitsführung aufbauten, die seinen Forderungen entspräche. Dies lässt ihn soweit gehen, 1933 zu sagen: «Der einzige politische Kopf Europas, der das gesamte Problem bis zu einem gewissen Grade überblickt und anzufassen weiss, ist Mussolini. ... Es ist fraglich, ob andere Völker zu einem faschistischen Experiment überhaupt fähig wären. Mussolini ist ein wirklicher Führer und sein Auftreten bedeutet eine neue Hoffnung für die ganze Menschheit» [39]. Bircher-Benner betont auch, dass ihm Mussolini einen Arzt zum Erlernen seiner Methoden schickt [12, S. 3 f]. Manche theoretisch-eugenische Formulie- rung fällt dabei schärfer aus als seine praktischen Forderungen oder Umsetzungen. Bircher-Benner scheint zwar in der Naturheilkunde ein Potential zur Stärkung der Konstitution zu sehen, wobei letztlich auch eugenische Aspekte anklingen, aber dabei geht er nicht so weit wie andere medizinische Autoren, z.B. Werner Kollath, für den die Naturheilkunde durch Verbesserung des Genotyps zur Rassenverbesserung beitragen könnte [14].

Sein Wunsch nach einer «Heilorganisation» lässt ihn seine Privatklinik errichten $[15$, S. 5; 12, S. 14]. Die staatliche Anerkennung und Beteiligung an einer Ärzteschule glaubte er eine Zeit lang am Rudolf-Hess-Krankenhaus in Dresden umsetzen zu können [14, 37, 40]; auch als es nicht dazu kommt, sieht er Deutschland weiterhin als beispielhaft an [7, S. 111].

Einen gewissen Gegensatz zu seinen Äusserungen zu faschistischen Regierungen stellt die Satzung der Kulturgesellschaft Zürich dar, die das Protektorat für das Volkssanatorium für Ordnungstherapie übernimmt: «Die KgZ steht auf schweizerisch demokratischem Boden und enthält sich jeder Art von parteipolitischen und konfessionellen Bindungen. Sie vertritt aber die Überzeugung, dass freiwillige Lebensordnung des Einzelnen in keiner Volksgemeinschaft so wichtig ist wie in der demokratischen...» Trotz dieser Aussage zur demokratischen Verankerung des Volkssanatoriums für Ordnungstherapie wird im Begleittext positiv von der «kräftigen Entwicklung» in Deutschland berichtet, wo «im Sinne einer gewandelten ärztlichen Weltauffassung, ... der ,neue Arzt' die Ordnungstherapie» lerne [29, S. 647]. Die Ordnungstherapie von Bircher-Benner korrespondiert aufgrund genealogischer Bezüge zur «Konstitutions- und Rassenverbesserung» mit Forderungen eugenischer Bewegungen der 30er Jahre.

\section{Ergebnisse}

Bircher-Benner prägte 1937 Wort und Inhalte der Ordnungstherapie. Im Gegensatz zu der heutigen Definition der Ordnungstherapie war sie bei Bircher-Benner nicht eine der fünf Bereiche der Naturheilkunde, sondern das übergeordnete naturheilkundliche Konzept, unter das er als Eklektiker andere Therapieverfahren ordnete. Bircher-Benners Konzeption der Ordnungstherapie liegt - trotz aller Parallelen zu bereits existierenden Gesundheitsvorstellungen in der Medizingeschichte, die auf Aspekten einer «Harmonie» im Organismus gründen - eine eigene nosologische Terminologie zugrunde: Unordnung im Leben bewirkt Krankheit in der Leib-Geist-SeeleEinheit. Durch die Vereinigung von Somatotherapie und Psychotherapie in der Ordnungstherapie sei eine Wiederherstellung der Ordnung und damit Gesundheit möglich, wenn noch «Naturheilkräfte» im Organismus angeregt werden könnten, die Erkrankung also nicht zu weit fortgeschritten sei. Es ist auffallend, dass Bircher-Benner unter dem Begriff der Somatotherapie zwar Ernährungslehre, Sonnen- und Lichttherapie, Hydrotherapie, Bewegungstherapie, Atmungstech- 
nik und Ordnung des Lebensrhythmus nennt, aber nur mit Vorbehalten die Phytotherapie. Sie und die Homöopathie verwendet er nach eigenen Aussagen nur selten. Der Ordnungsgedanke ist bei Bircher-Benner nicht nur medizinisch-physiologisch geprägt, sondern auch von gesellschaftspolitischen Ordnungsvorstellungen eugenischer Bewegungen.

\section{Diskussion}

Der Begriff der Ordnungstherapie wurde lange Zeit als eine der fünf Säulen der Kneipp-Therapie bezeichnet, obwohl er nicht von Kneipp geprägt oder dezidiert verwendet wurde. Gelegentlich finden sich bei den entsprechenden Autoren Hinweise auf folgende Textstelle bei Kneipp: «Denn wie Viele waren hier, die nach langem Gebrauche der Wasserkur nicht besser dran waren, und bei denen die neurasthenischen Schmerzen an allen möglichen Stellen sich immer wieder fühlbar machten! Die Betroffenen konnten nicht schlafen und nicht essen; sie verfielen in Melancholie, und erst als man den Zustand ihrer Seelen kannte und da Ordnung hineinbrachte, ging es mit dem körperlichen Leiden auch besser. Sie bekamen mehr Ruhe, mehr Zufriedenheit, kurz, sie fühlten sich glücklicher» [41]. Der erste Satz der Auflage von 1897 gibt hier einen wichtigen Hinweis auf den Kontext der Aussage, nämlich das Kapitel «Nervosität, Neurasthenie und Nervenschwäche» in Kneipps Buch. Kneipp gibt uns als Therapeut den Hinweis, dass er nicht alle Erkrankungen als rein körperlich bedingt ansieht. Dies wäre ein Indiz für ein weitblickendes medizinisches Verständnis. Da Kneipp Geistlicher war, ist es aber auch nachvollziehbar, dass er aus religiösen Gründen von der Ordnung in der Seele, im Sinne der Erlösung oder Rettung einer Seele spricht. Aus diesem Kontext aber die Prägung der Ordnungstherapie abzuleiten scheint nicht gerechtfertigt [42, 43]. Vielmehr muss die Nennung des Begriffes Ordnungstherapie durch Kneipp-Ärzte im 20. Jh. als eine nachträgliche Interpretation oder Weiterentwicklung der Kneipp-Therapie angesehen werden.

So wurde im Jahr 1947 der Kneippärztebund neu gegründet [44, S. 703], und Schwerpunkt seiner ab 1949 durchgeführten Lehrgänge war die Hydrotherapie [45]. Einer der bekanntesten Kneipp-Ärzte, Christian Fey, verzichtet in Schriften in den 1950er Jahren mal auf den Begriff «Ordnungstherapie» [46, 47], mal spricht er sogar von «Lebensordnungstherapie» [48]. Die Kneipp-Ärzte sehen zu dieser Zeit die Ordnungstherapie nicht einheitlich als eine der fünf Säulen der Kneipp-Therapie an. So finden sich z.B. Angaben zu «vier tragenden Säulen ... der Naturheilkunde Kneippscher Prägung ...: 1. die Hydrotherapie, 2. die Diätetik, 3. die Phytotherapie, 4. die Psychotherapie» [49, S. 2; s.a. 50, S. 885]. Allerdings wird ab den $1970 \mathrm{er}$ Jahren fast durchgehend von «fünf Säulen» [51-53] oder «Wirkprinzipien der Kneipp-Therapie» [5, 54, 55, 69] gesprochen und der Begriff Ordnungstherapie dazugezählt. Mit der Formulierung von «fünf Säulen der Naturheilkunde nach
Kneipp» seit den 1990er Jahren erreichte diese Tradierung mehr oder weniger definitorischen Charakter für das Fach Naturheilkunde, trotz inhaltlicher Unterschiede hinsichtlich der Ordnungstherapie [1].

Zwischenzeitlich gab es auch Versuche, die Ordnungstherapie nach Bircher-Benner zu erweitern. So stellt Heinz Bottenberg 1948 die Blutegelbehandlung am Ende seines gleichnamigen Buches explizit in den Kontext der «Ordnungstherapie nach Bircher-Benner», auch wenn er einräumt, dass sie «die tieferen Ursachen» im Allgemeinen nicht berührt [56]. Eine andere Interpretation schlägt G. Bachmann vor, der 1959 die Akupunktur selbst als Ordnungstherapie bezeichnet [57].

Seit den 1990er Jahren löst sich die Rezeption des Begriffes Ordnungstherapie von der Prägung durch Bircher-Benner, wie an den Ausführungen von Weber und Dogs ersichtlicht wird. Der Erste konstatiert zwar, dass «sich kaum jemand finde, der präzise zu definieren vermag, was die Ordnungstherapie alles beinhalte», dennoch sei sie «ein übergeordnetes Prinzip der Naturheilverfahren» [58, S. 15 f]. Er zählt dann z.B. strukturierte Körpertherapie, Feldenkrais, Atemtherapie, autogenes Training, Realtherapie und systemische Familientherapie dazu. Dogs zählt unter dem Begriff «dynamische Psychotherapie» ähnliche Gruppen auf (z.B. autogenes Training, Meditation) [6, S. 296].

Auch Hörning resümiert 1999, die Ordnungstherapie sei «unklar definiert» und beschreibt dann drei Ebenen, physisch, psychisch, sozial, die das Gebiet umfassten [59]. Moerchel erwähnt Ordnungstherapie, in dem von ihm 1989 herausgegebenen Lexikon der Naturheilkunde von Krug nicht, und stellt sie 2002 in den Kontext von Kneipp [60, 61]. Melchart et al. setzen den Begriff der Ordnungstherapie seit einiger Zeit wieder mit einem umfassenden Konzept eines pädagogischen strukturierten Gesundheitstrainings gleich, das [62-64] mehr dem Selbstverständnis «hedonistisch» orientierter westlicher Industriegesellschaften entspricht.

Die ordnungspolitischen Aspekte der Ordnungstherapie sind historisch gesehen nicht von der Hand zu weisen, und der erzieherische Impetus scheint aufgrund des sich selbst beschränkenden Moments wenig attraktiv. Vermutlich werden deshalb einige Aspekte, die im deutschsprachigen Raum ordnungstherapeutische Tradition haben, in angloamerikanischen Ländern zunehmend unter anderen Begriffen berücksichtigt, wie z.B. life style modification, life style management, life style intervention oder body mind intervention [65-68]. Die mitunter inhaltlichen Parallelen zu einzelnen Aspekten der traditio nellen Ordnungstherapie (z.B. Ernährung, Bewegung und Psychotherapieformen im weitesten Sinne) werden hierbei aber meist in einen weitläufigen interdisziplinären Kontext gestellt (Fitness-Wellness, Psycho-Neuro-Immunologie).

Prägung und Definition der Ordnungstherapie durch BircherBenner verdeutlichen, dass ihre Zuschreibung als eine der fünf Säulen der originären Kneipp-Therapie überdacht werden sollte, unbeschadet der Verdienste der teilweise intensiven Diskussion der 1980er Jahre [69]. Den vielfältigen Inter- 
pretationen durch spätere Autoren ist gemeinsam, dass sie die Ordnungstherapie nicht als ein umfassendes Therapiekonzept (Somato- und Psychotherapie) wie Bircher-Benner verstehen, sondern, von einzelnen Ausnahmen abgesehen [62, 70], als einen von fünf Teilbereichen in der Naturheilkunde. Oft werden darunter ganz unterschiedliche therapeutische Ansätze subsumiert, ohne Hinweis darauf, dass sie sich vom umfassenden Konzept von Bircher-Benner entfernen und eigene Auffassungen darstellen. Die Ordnungstherapie nach BircherBenner ist ein mehrdimensionaler Ansatz (z.B. Gleichzeitigkeit von Somatotherapie und Psychotherapie). Diese Mehr- dimensionalität kann wesentliche praktische Momente einer von Patienten erwarteten ganzheitlichen Therapie umfassen. Jedoch unter der Voraussetzung, dass Ganzheitlichkeit mit einem komplexen, für den Patienten frei wählbaren Therapieansatz zu seinem Wohle gleichgesetzt wird.

\section{Dank}

Wir danken den MitarbeiterInnen des Bircher-Benner-Archivs am Medizinhistorischen Institut Zürich, besonders Eberhard Wolff und Sonja Furger, für den Zugang zu Archivalien der Bircher-Benner-Privatklinik.

\section{Literatur}

1 Bühring M: Naturheilmittel, Naturheilverfahren, Naturheilkunde - eine Einführung; in Bühring M (Hrsg): Naturheilverfahren und unkonventionelle Medizinische Richtungen. 32. Aufl., Berlin, Springer, 2003, S. 1-29.

2 Melchart D: Was versteht man unter «Naturheilverfahren» in Deutschland? in Melchart D, Brenke R, Dobos G, Gaisbauer M, Saller R (Hrsg): Naturheilverfahren. Leitfaden für die ärztliche Aus-, Fort- und Weiterbildung. Stuttgart, Schattauer, 2002, S. 3-8.

3 Ärztekammer Berlin: Weiterbildungsordnung der Ärztekammer Berlin. Berlin, Ärztekammer Berlin, 2000, S. 113-114.

4 Wilhelm R: Ordnungstherapie; in Schimmel KC (Hrsg): Lehrbuch der Naturheilverfahren. 2. Aufl., Bd. 1, Stuttgart, Hippokrates, 1990, S. 473-482.

5 Penning W: Ordnungstherapie; in Dittmar FW, Loch EG, Wiesenauer M (Hrsg): Naturheilverfahren in der Frauenheilkunde und Geburtshilfe. Stuttgart, Hippokrates, 1994, S. 18-27.

6 Dogs W: Ordnungstherapie; in Hentschel HD (Hrsg): Naturheilverfahren in der ärztlichen Praxis 2. Aufl., Köln, Deutscher Ärzte Verlag, 1996, S. 296-317.

7 Bircher-Benner M: Vom Werden des neuen Arztes. Erkenntnisse und Bekenntnisse. Dresden, Heyne, 1938.

8 Kollenbach D: Maximilian Oskar Bircher-Benner. Krankheitslehre und Diätetik. Med. Diss. Köln, 1974.

9 Prospekt von Dr. Bircher-Benners Sanatorium «Lebendige Kraft» Zürich. Prospekt der Klinik. n. p. n. d. ,ca. 1906, S. 22 f.

10 Bircher-Benner M: Grundzüge der ErnährungsTherapie. 3. Aufl., Berlin, Salle, 1909, S. 73.

11 Bircher-Benner M: Food Science for All and a New Sunlight Theory of Nutrition. ed 2, London, Daniel Company, 1939, p 112.

12 Bircher-Benner M: The Prevention of Disease by Correct Feeding. London, Food Education Society, [1933], p 14.

13 Bircher-Benner M: Vom Wesen und der Organisation der Nahrungsenergie (= Kleine HippokratesBücherei. Bd. VIII) Stuttgart, Hippokrates, 1936, S. 20

14 Melzer J: Vollwerternährung: Diätetik, Nationalsozialismus, sozialer Anspruch. Stuttgart, Steiner, 2003

15 Bircher-Benner M: Vom Sinn einer therapeutischen Organisation. Leipzig, Hippokrates, 1935

16 Bircher-Benner Archiv Zürich, Patientenakten, Journal-Nr. 397, Kurvorschriften 1925.

17 Bircher-Benner M: Seelenbehandlung. Wendepunkt 1933;10:237-246.
18 Bircher R: Bircher-Benner. Kurzer Überblick über Lebenslauf und Lebenswerk. Wendepunkt 1937;14: 471-509.

19 Bircher-Benner M: Mein Dankeswort. Wendepunkt 1937; 14:523-529.

20 Sihle M: Über das Weltbild des Arztes und den Sinn der Krankheit. Ein Appell zur Lebenssynthese. Wien, Weidmann, 1934, S. $101 \mathrm{f}$

21 Bircher-Benner M: Über das Weltbild des Arztes und den Sinn der Krankheit. Inhaltsübersicht des unter diesem Titel erschienenen Buches von Prof. Dr. Martin Sihle. Zürich, Wendepunkt, 1935.

22 Bircher-Benner M: Fragen des Lebens und der Gesundheit. Zürich, Wendepunkt, 1937.

23 Bircher-Benner M: Vom Heilen und Verhüten. Wendepunkt 1937;14:7-14.

24 Bircher-Benner M: Ein Wendepunkt im Kampfe gegen den Krebs. Wendepunkt 1937;14:241-244.

25 Bircher R: Bircher-Benner. Kurzer Überblick über Lebenslauf und Lebenswerk. Zum 70. Geburtstag. Wendepunkt 1937;14:471-511.

26 Bircher R: Leben und Lebenswerk. Bircher-Benner. Zürich, Bircher-Benner, 1959, S. 125.

27 Kollenbach D: Maximilian Oskar Bircher-Benner Krankheitslehre und Diätetik. Med. Diss. Köln, 1974, S. 167.

28 Anonym: Zürcher Kulturgesellschaft. Wendepunkt 1935;12:274-275.

29 Anonym: Kulturgesellschaft Zürich (KgZ). Wendepunkt 1938;15:646-648.

30 Anonym: Neues vom Volkssanatorium für Ordnungstherapie. Wendepunkt 1938;15:354-356.

31 Anonym: Zuwachs zum Freibettfonds des Volkssanatoriums für Ordnungstherapie. Wendepunkt 1939;16:235.

32 Bircher-Benner M: Mitteilungen betr. Volkssanatorium für Ordnungstherapie. Wendepunkt 1938;15: 105-106.

33 Bircher FE: Diät und Behandlung im Volkssanatorium für Ordnungstherapie. Übersicht über die ersten 100 Kuren. Zürich, Ostheim, 1940.

34 Bircher W: Herzkrankheiten und ihre Heilung unter dem Gesichtspunkt der Volldiagnose und der Ordnungstherapie. Zürich, Wendepunkt, [1943]

35 Bircher W: Hautkrankheiten und ihre Heilung unter dem Gesichtspunkt der Volldiagnose und der Ordnungstherapie. Zürich, Wendepunkt, [1942].

36 Liechti von Brasch D, Kunz-Bircher A: 70 Jahre Erfahrungsgut der Bircher-Benner Ordnungstherapie. Erfahrungsheilkunde 1970;14:181-186, 229237, 262-273

37 Wirz A: Die Moral auf dem Teller. Zürich, Chronos, 1993.

38 Melzer J: Werner Kollath 1933-1945. Dokumentensammlung und Kommentare. Bad Soden, Wernerund-Elisabeth-Kollath-Stiftung, 2002, S. 5-7.
39 Bircher-Benner M: Der Menschenseele Not, 2. Teil. Zürich, Wendepunkt, 1933, S. 72.

40 Lienert M: Das Stadtkrankenhaus Dresden Johannstadt zur Zeit des Nationalsozialismus; in Scholz A, Heidel CP, Lienert M (Hrsg): Vom Stadtkrankenhaus zum Universitätsklinikum. 100 Jahre Krankenhausgeschichte in Dresden. Köln, Böhlau, 2001, S. 105-142.

41 Kneipp S: Codizill zu meinem Testamente für Gesunde und Kranke. 3. Aufl., Kempten, Kösel, 1897, S. 282-283.

42 Machens R: Ordnungstherapie; in Augustin M, Schmiedel V (Hrsg): Praxisleitfaden Naturheilkunde. Neckarsulm, Jungjohann, 1994, S. 162-166.

43 Schmiedel V: Ordnungstherapie; in Augustin M, Schmiedel V (Hrsg): Leitfaden Naturheilkunde. 4. Aufl., München, Urban und Fischer, 2003, S. $428-438$

44 Hoff A: Der Kneipparzt. Hippokrates 1963;34:703706.

45 Fey C: Die Entwicklung des Kneippärztebundes e.V. Ärztliche Gesellschaft für Hydrotherapie, Physiotherapie. Hippokrates 1959;30:69-70.

46 Fey C: Entwicklung und Fortschritt des Kneippschen Heilverfahrens; in Ergebnisse der physikalisch-diätetischen Therapie. Bd. 3., Dresden, 1948 , S. 113.

47 Fey C: Kneippkur richtig durchgeführt. Bad Wörishofen, Verlag für ärztliche Fortbildung, 1952.

48 Fey C: Kneipptherapie bei Herz-Kreislaufstörungen. Bad Wörishofen, Verlag für ärztliche Fortbildung, 1952, S. 7

49 Kaiser JH: Gesichtspunkte für die Durchführung der Kneipptherapie in Klinik und Praxis. Hydrother Physiother 1955;1:2-7.

50 Koeppen S, Kostka F: Die Kneipp-Therapie in der internen Klinik. Hippokrates 1959;30:884-887.

51 Schumacher-Wandersleb O: Möglichkeiten der modernen Kneipp-Therapie bei Erkrankungen des Herz-Kreislauf-Systems. Krankenpflege 1977;31: 364-365.

52 Uehleke B: A. Kneipptherapie - Geschichte un Entwicklung; in Zentrum zur Dokumentation für Naturheilverfahren, Forschungsinstitut Freie Berufe (Hrsg): Dokumentation der besonderen Therapierichtungen und natürlichen Heilweisen in Europa. Ganzheitliche Medizinsysteme. Bd. 1. Halbband 1. In der Kostenerstattung anerkannte Medizinsysteme. Essen, VGM Verlag, 1991, S. 765-767.

53 Müller-Limmroth W: B. Die fünf Säulen der Kneipp-Therapie. Ordnungstherapie; in Zentrum zur Dokumentation für Naturheilverfahren, Forschungsinstitut Freie Berufe (Hrsg): Dokumentation der besonderen Therapierichtungen und natürlichen Heilweisen in Europa. Ganzheitliche Medizinsysteme. Bd. 1. Halbband 1. In der Kostenerstattung anerkannte Medizinsysteme. Essen, VGM Verlag, 1991, S. 767-781. 
54 Brüggemann W: Einleitung; in Brüggemann W (Hrsg): Kneipptherapie. Ein Lehrbuch. Berlin, Springer, 1980, S. 1-4.

55 Günther R, Jantsch H: Physikalische Medizin. Berlin, Springer, 1982, S. 200.

56 Bottenberg H: Die Blutegelbehandlung. 2. Aufl., Stuttgart, Hippokrates, 1948, S. 213-215.

57 Bachmann G: Die Akupunktur, eine Ordnungstherapie. Ulm, Haug, 1959.

58 Weber K (Hrsg): Kurs Naturheilverfahren: Ordnungstherapie. Stuttgart, Sonntag, 1995.

59 Hörning M: Die drei Ebenen der Ordnungstherapie. Ärztez Naturheilverfahren 1999;40:608-616.

60 Krug E: Lexikon der Naturheilkunde (Hrsg. v. Moerchel J). Heidelberg, Haug, 1989.

61 Moerchel J: Ordnungstherapie = Ordnung des Seins. Aachen, Shaker, 2002.

62 Melchart D, Döbrich R, Ebner K, Grenz M, Heimerl K, Kaupp H, Nagel R, Paul A, Schöngart S, Steinhardt H, Wunderlich S: Strukturiertes Gesundheitstraining als naturheilkundliche Ordnungstherapie. Prävention 1999;22:46-49.

63 Melchart D: Konzeption einer modernen Gesundheitspädagogik; in Melchart D, Brenke R, Dobos G, Gaisbauer M, Saller R (Hrsg): Naturheilverfahren. Leitfaden für die ärztliche Aus-, Fort- und Weiterbildung. Stuttgart, Schattauer, 2002, S. 5563.

64 Wunderlich S, Melchart D: Strukturiertes Gesundheitstraining in der klinischen Praxis; in Melchart D, Brenke R, Dobos G, Gaisbauer M, Saller R (Hrsg): Naturheilverfahren. Leitfaden für die ärztliche Aus-, Fort- und Weiterbildung. Stuttgart, Schattauer, 2002, S. 74-88.

65 Gohlke H: Prävention durch Lebensstiländerung: Was ist gesichert? Herz 2004;29:139-144.
66 Lear SA, Ignaszewski A, Linden W, Brozic A, Kiess M, Spinelli JJ, Haydn Pritchard P, Frohlich JJ: The Extensive Lifestyle Management Intervention (ELMI) following cardiac rehabilitation trial. Eur Heart J 2003;24:1920-1927.

67 Harris SB, Petrella RJ, Leadbetter W: Lifestyle interventions for type 2 diabetes. Relevance for clinical practice. Can Fam Physician 2003;49:16181625.

68 Rybarczyk B, DeMarco G, DeLaCruz M, Lapidos S, Fortner B. A classroom mind/body wellness intervention for older adults with chronic illness: comparing immediate and 1-year benefits. Behav Med 2001;27:15-27.

69 Brüggemann W (Hrsg): Würzburger Gespräche über die Kneipptherapie. Bd. 5 Ordnungstherapie. Bad Wörishofen, Sebastian-Kneipp-Zentral-Institut, 1980.

70 Das S: Ordnungstherapie; in Bachmann R, Saller R (Hrsg): Naturheilverfahren in der Praxis. Ballingen, Spita, 1998, 5/3.1, S. 1-3; 5/3.4, S. 1-14.

71 Bachmann RM: Naturheilverfahren für die ärztliche Praxis. Bd. 1 Methoden. Erlangen, perimed, 1989, S. 78.

72 Brüggemann W: V. Ordnungstherapie; in Zentrum zur Dokumentation für Naturheilverfahren, Forschungsinstitut Freie Berufe (Hrsg): Dokumentation der besonderen Therapierichtungen und natürlichen Heilweisen in Europa. Ganzheitliche Medizinsysteme. Bd. 1. Halbband 1. In der Kostenerstattung anerkannte Medizinsysteme. Essen, VGM Verlag, 1991, S. 826-829.
73 Hentschel HD (Hrsg): Naturheilverfahren in der Ärztlichen Praxis. Köln, Deutscher Ärzte-Verlag, 1991.

74 Harth V: Praxis der Naturheilverfahren. Stuttgart, Hippokrates, 1992.

75 Bachmann RM: Praxisservice Naturheilverfahren. Stuttgart, Hippokrates, 1996.

76 Hentschel HD: Einleitung; in Hentschel HD (Hrsg): Naturheilverfahren in der ärztlichen Praxis. 2. Aufl., Köln, Deutscher Ärzte Verlag, 1996 , S. $23-42$.

77 Schmiedel V, Augustin M: Handbuch Naturheilkunde. Heidelberg, Haug, 1997.

78 Pschyrembel W: Ordnungstherapie. Klinisches Wörterbuch. 258. Aufl., Berlin, de Gruyter, 1998.

79 Psychrembel W: Ordnungstherapie. Wörterbuch Naturheilkunde. 2. Aufl., Berlin, de Gruyter, 2000.

$80 \mathrm{Schmiedel} \mathrm{V:} \mathrm{Ordnungstherapie;} \mathrm{in} \mathrm{Augustin} \mathrm{M,}$ Schmiedel V (Hrsg): Leitfaden Naturheilkunde. 4. Aufl., München, Urban und Fischer, 2003, S. 428438

81 Bircher-Benner Archiv Zürich, Patientenakten, Journal-Nr. 165, 308, 380, 397, 460, 503, 517, 714, 589, 698, 1213, 1314, 1377, 1495, Verordnungen

82 Bircher-Benner M: Ordnungsgesetze des Lebens als Wegweiser zur echten Gesundheit. Unveränderte Neuausgabe, Bad Homburg v.d.H., Bircher-Benner, 1984.

83 Bircher-Benner M: Das Volkssanatorium für Ordnungstherapie. 2. Aufl., Zürich, 1938. 